This item was submitted to Loughborough's Institutional Repository (https://dspace.lboro.ac.uk/) by the author and is made available under the following Creative Commons Licence conditions.

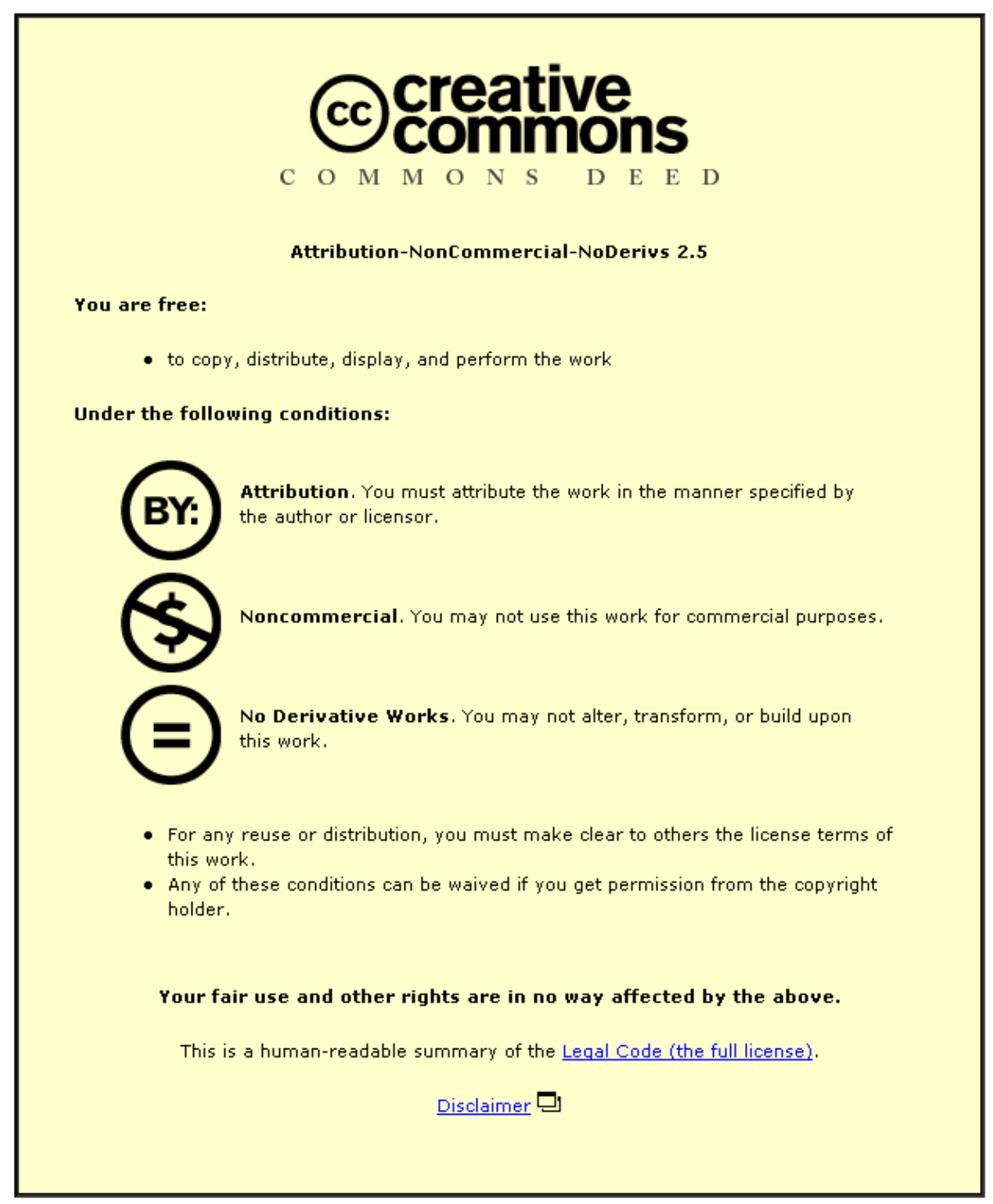

For the full text of this licence, please go to: http://creativecommons.org/licenses/by-nc-nd/2.5/ 


\title{
Parametric Data-Parallel Architectures for TLM Acceleration
}

\author{
Vassilios A Chouliaras James A Flint Yibin Li \\ V.A.Chouliaras@Iboro.ac.uk J.A.Fint@lboro.ac.uk Y.Li2@lboro.ac.uk \\ Department of Electronic and Electrical Engineering, Loughborough University, \\ Ashby Road, Loughborough, Leicestershire, LE11 3TU, UK. \\ Tel: +44 1509227113 , Fax: +44 1509227014
}

\begin{abstract}
We discuss the architecture and microarchitecture of a scalable, parametric vector accelerator for the TLM algorithm Architecturelevel experimentation demonstrates an order of magnitude complexity reduction for vector lengths of 16 32-bit single-precision elements. We envisage the proposed architecture replicated in a SoC environment thus, forming a multiprocessor system capable of tapping parallelisin at the thread level as well as the data levet.
\end{abstract}

\section{Introduction}

Prior attempts to implement the TLM algorithm [1] on general-purpose architectures have fallen into two major categories: Shared memory, cache coherent multi-processors $[2,3]$ and distributed processors [4] with shared- memory machines often demonstrating better performance.

The TLM is a highly-parallel three-dimensional numerical algorithri which has the potential for being accelerated along its innermost loop via vectorization thus, tapping parallelism at the data level (DLP). Furthermore, the algoritbm can be statically 'sliced' (threaded) along the second outer loop, and be executed on the previously mentioned plafforms via different processors executing different iterations. Such parallelism is known as thread-level-parallelism [5] and is currently being pursued by all major microprocessor vendors.

Successful acceleration of such parallel codes depends very much on the algorithmic communication pattern which dictates the level of data sharing across the multiple processors. In the case of the TLM, data transfers between individual nodes is very high and in extreme cases the data transfer during the connect part of the algorithm can be much more computationally expensive than the numerical calculations during scattering. The performance differential between shared memory and distributed machines is often attributed to such data sharing issues.

Custom architectures for accelerating TLM codes have been proposed in the past by Stothard and Pomeroy [6]. Our work proposes a custom vector approach to accelerating the inner loop of TLM codes, quite unlike this earticr work. In our case, an embedded 32-bit processor is augmented with a configurable, extensible custom vector accelerator and resides on an on-chip-bus [7] thus, forming a finely-tuned SoC computation kernel for the TLM algorithm.

\section{Vector architecture}

The programmer's model of the parametric vector accelerator for TLM is depicted in Figure 1:

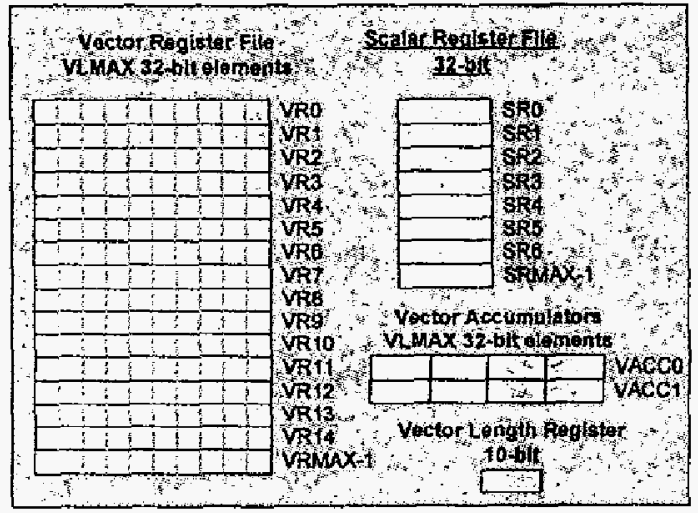

Figure 1: Vector accelerator programmer's model

The programmer's model specifies a parametric number of vector registers (VRMAX), each consisting of a parametric number of 32-bit singleprecision elements (VLMAX). There is a scalar register file consisting of a parametric number of scalar 32-bit elements (SRMAX), used for virtual address computation, immediate passing and vector splat operations. Additionally, there are two vector accumulators each holding VLMAX single-precision elements and finally, the vector length register (VLEN) which specifies the number of bytes that will be affected by the currently executing vector opcode. The Instruction Set Architecture (ISA) of the accelerator includes standard vector floating point operations except division, vector Load/Stores, and a generalized permute instruction. A large number of sub-element manipulation instructions (including vector splat instructions) can be synthesized based on the three-operand permute infrastructure. The ISA is summarized in Table 1 
Table 1: Vector Copmcessor ISA

\begin{tabular}{|c|c|}
\hline Instruction & Description \\
\hline MVSR2VLEN & $\begin{array}{l}\text { Transfer scalar register to vector } \\
\text { length register (VLEN) }\end{array}$ \\
\hline MVSR2CSR & $\begin{array}{l}\text { Transfer RISC scalar register to } \\
\text { coprocessor scalar register }\end{array}$ \\
\hline MVCSR2R & $\begin{array}{l}\text { Transfer coprocessor scalar register } \\
\text { to RISC register }\end{array}$ \\
\hline MVSR2CVEL & $\begin{array}{l}\text { Move RISC scalar register to } \\
\text { coprocessor vector element }\end{array}$ \\
\hline MVCVEL2R & $\begin{array}{l}\text { Move coprocessor vector element } \\
\text { to RISC scalar register }\end{array}$ \\
\hline VLDU & $\begin{array}{l}\text { Load vector register unaligned } \\
\text { under VLEN }\end{array}$ \\
\hline VSTU & $\begin{array}{l}\text { Store vector register unatigned } \\
\text { under VLEN }\end{array}$ \\
\hline VPERM & $\begin{array}{l}\text { Three-operand bytewise vector } \\
\text { permute }\end{array}$ \\
\hline VSPLAT & $\begin{array}{l}\text { Splat coprocesson scalar register to } \\
\text { coprocessor vector regitoter }\end{array}$ \\
\hline VFPADD.S & $\begin{array}{l}\text { Vector floating-point add (single } \\
\text { precision) under VLEN }\end{array}$ \\
\hline VFPSUB.S & $\begin{array}{l}\text { Vector flowating-point ab (sinde } \\
\text { poction) under VLN }\end{array}$ \\
\hline VFPMUL.S & $\begin{array}{l}\text { Vector floating-point mult (single } \\
\text { precision) under VLEN }\end{array}$ \\
\hline VFP & $\begin{array}{l}\text { Vector floofing point mul } \\
\text { fecumalate }\end{array}$ \\
\hline
\end{tabular}

\section{Vector microarchitecture}

The proposed' vector extensions are implemented as a tightly-coupled vector accelerator attached to an open-source, configurable, extensible, Sparc V8compliant RISC CPU [8]. The processor/coprocessor combination communicates via the AHB On-Chip Bus to the SDRAM controller which controls the offchip SDRAM part. A bigh level schematic of the scalar processor and vector accelerator is depicted in Figure 2.

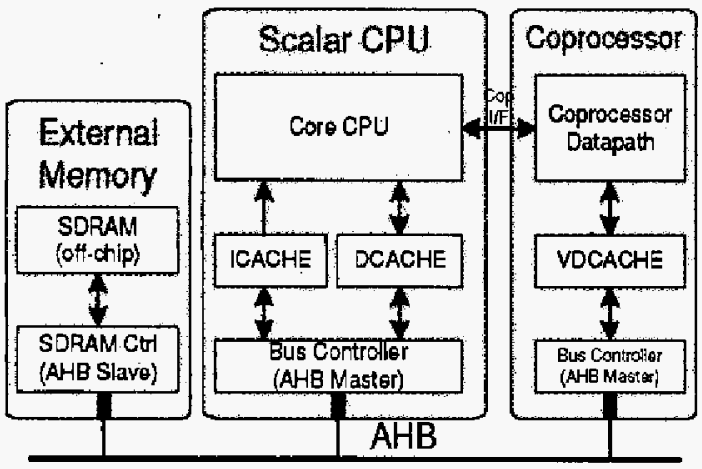

Figure 2: TLM computation kernel

As shown in the figure, there exists a bidirectional communication channel across the scalar processor and the vector accelerator. Though the open source
CPU provides a coprocessor interface, it was decided to implement that channel in order to ensure pipelined, lockstep operation of the accelerator and timely transfer of data to and from the main CPU. Typical transactions on the developed channel are depicted in Figure 3.

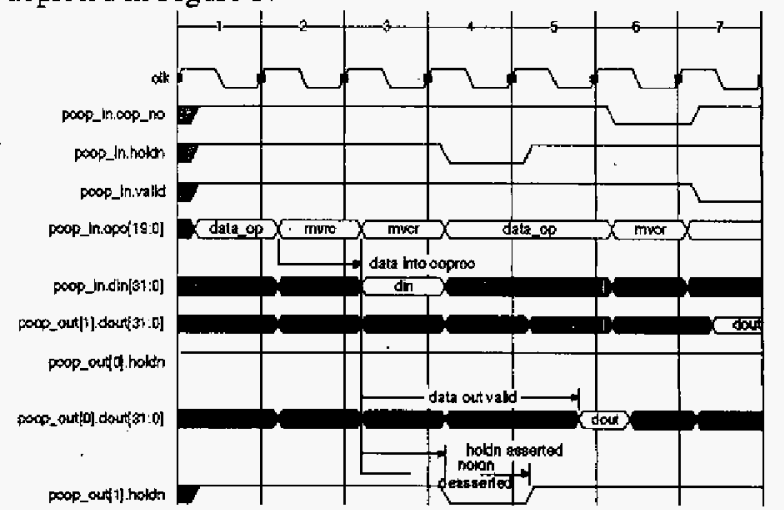

Figure 3: Processor-Coprocessor communication channel

The detailed microarchitecture of the combined scalar processor/vector coprocessor for a vector length of two 32-bit (single-precision) elements is depicted in Figure 4.

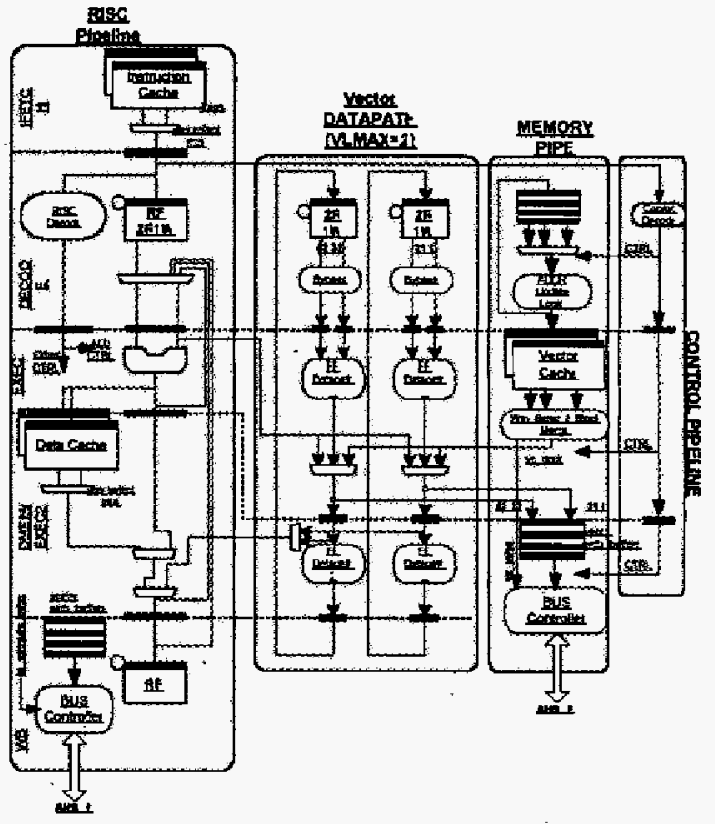

Figure 4: Detailed microarchitecture

Instructions are fetched from the multi-way setassociative instruction cache and stored in a single 32-bit register. Typically, high-performance RISC processors of equal pipeline depth would extract the source operand fields right after instruction cache access and set up the synchronous register file. Unfortunately, that is not the case in the particular processor which, due to the windowing scheme of the Sparc V8 architecture, requires access to the current- 
window-pointer (CWP) register in order to compute a physical register file address. As a result, source operand addresses are set-up on the falling edge of the clock in the DECODE stage. During this stage, the register file is accessed and the two source registers are retrieved. Operand bypassing takes then place and the resolved operands are clocked into the ALU input registers, ready for execution. It is during this stage that the vector opcodes are identified and dispatched to the tightly-coupled vector accelerator. Decoding logic in the later produces a number of control fields which are pipelined down the control pipeline. Vector operand accesses are triggered by the falling edge of the clock curing decode, for reasons of symmetry to the scalar pipeline.

During the EXEC stage, the RISC CPU executes the scalar instruction or computes the virtual address of a Load/Store operation. In the same stage, the vector accelerator performs the first stage of the pipelined floating point computations. In the next stage, scalar data return to the main processor via the data cache return path whereas the vector accelerator performs the last stage of execution. Due to the very tight timing constraints, floating point results are stored in an intermediate register prior to committing to the vector register file.

\section{Methodology}

We have applied a basic implementation of the SCN TLM algorithm [1] in which no external boundary conditions were used. In the particular case, a single output node was used as a diagnostic aid to verify correct operation. We used the accelerated scatter method of Naylor and Ait-Sadi as proposed in [9].

The non-vectorized (scalar) algorithm was profiled both in native mode (IA32 Linux) as well as on our simulated processor for consistency of results. Scalar code profiling revealed a sçatter:connect complexity ratio of 63:37, averaging over all the studied configurations.

Our simulation infrastructure is based around the simplescalar toolset [10] which provides a complete. computer architecture modelling and performance evaluation environment. The compiler used was GCC 2.7.3 with optimizations (-O3).

\section{Results}

The reference problem chosen for benchmarking was a fixed mesh of $10^{6}$ nodes. This number is convenient as it gives a prime factorisation of $2^{6} \times 5^{6}$, which allows for the aspect ratio of the problem space to be varied over a reasonable range whilst maintaining the same number of nodes.

We measured the absolute complexity (dynamic instruction count) of the scalar code for all configurations of interest. Then, the vectorized code was run and its complexity recorded for a maximum vector length of up to 16 single-precision elements. Figures 5 and 6 depict the normalized complexity of the vectorized algorithm over maximum vector length.

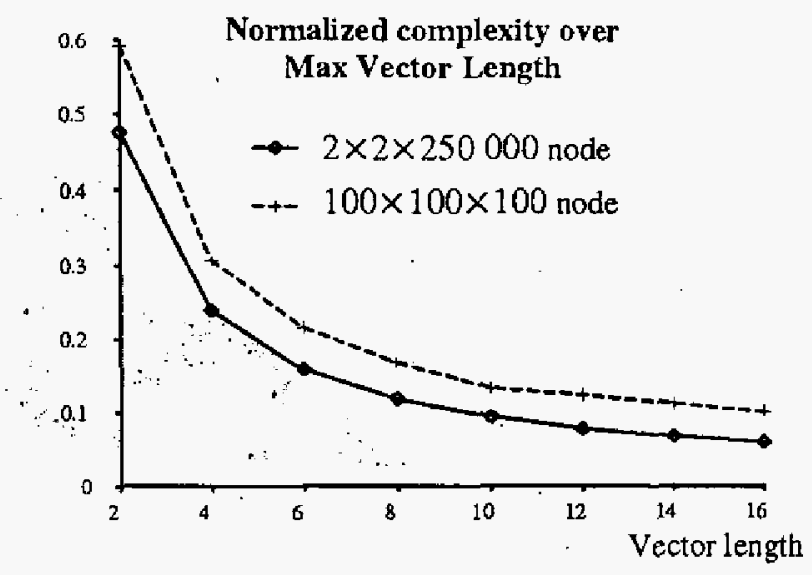

Figure 5 - Benchmarking using a thin and a cubic prablem space.

Figure 5 suggests that the optimal (less complex) configuration is where the problem space is thin, i.e. where the vector length is maximised.

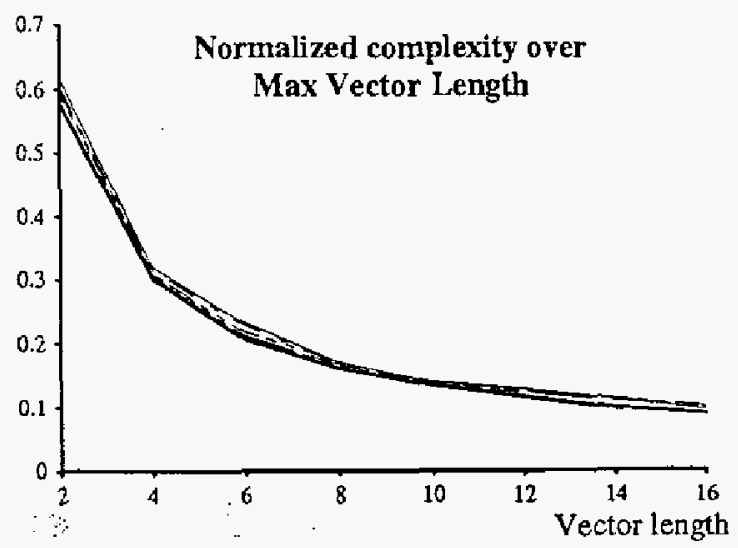

Figure 6-Iteration time for $80 \times 100 \times 125$ node mesh with differing alignment relative to the vector direction. All of these results show a similar speedup

Figure 6 depicts a $80 \times 100 \times 125$ configuration compared with a mesh of $100 \times 125 \times 80$, $80 \times 125 \times 100$, etc. These mesh dimensions were chosen as being typical of realistic model of an electromagnetic scattering situation. Results demonstrate that vectorization alignment changes only slightly the complexity (and hence run time) in all configurations. A vector length of 16 singleprecision elements showed a speedup of approximately an order of magnitude thus clearly demonstrating the benefit of using parallelism at the data level. 


\section{Conclusions}

We have proposed a parametric vector accelerator to exploit the significant amount of data level parallelism which is inherent within the TLM code. Our results demonstrate an order-of-magnitude performance improvement can be achieved for a vector length of 16 single-precision elements. Such a configuration is realizable with current VLSI technology. We are also actively investigating thread-level parallelism as the second major source of parallelism in the workload. Our scalable architecture can be replicated thus, creating a cachecoherent, embedded multiprocessor for TLM acceleration providing further performance benefits.

\section{References}

[1] P. B. Johns, "A symmetrical condensed node for the TLM method", IEEE Trans. Microw. Theory Tech., vol. 35, no. 4, pp. 370-377, 1987.

[2] J. L. Dubard, O. Benevello, D. Pompei, I. Le Roux, P. P. M. So, and W. J. R. Hoefer, "Acceleration of TLM through signal processing and parallel computing", in Computation in Electromagnetics, pp. 71-74, IEE, 25-27 November 1991.

[3] C. C. Tan and V. F. Fusco, "TLM modelling using an SIMD computer", Int. J. Numerical Modelling: Electronic Networks, Devices and Fields, vol. 6, pp. 299-304, 1993.

[4] P. J. Parsons, S. R. Jagues, S. H. Pulko, and F. A. Rabhi, "TLM modeling using distributed computing", IEEE Microw. and Guided Wave Lett, vol. 6, no. 3, pp. 141-142, 1996.

[5] J. Henessy, D. A. Patterson "Computer architecture: A quantitative approach", Morgan Kaufmann publishers, ISBN 1-55860-329-8

[6] D. Stothard and S. C. Pomeroy, "Dedicated TLM array processor", Applied Computational Electromagn. Soc. J., vol. 13, no. 2, pp. 188$196,1998$.

[7] "AMBA Specification (Rev 2.0)", www.arm.com

[8] "The Leon-2 processor User's manual, XST edition, ver. 1.0.14", http://www.gaisler.com

[9] P. Naylor and R. Ait-Sadi, "Simple method for determining 3-D TLM nodal scattering in nonscalar problems", Electron. Lett., vol. 28, no. 25 , pp. 2353-2354, 1992.
[10] D. Burger, T. Austin, 'Evaluating Future Microprocessors: The Simplescalar Tool Set', http://www.simplescalar.com 